\title{
Investigation on crystal structure, third-order nonlinear optical and thermal studies of 4-nitroanilinium 5-sulfosalicylic acid monohydrate single crystal
}

\author{
P.K. Sivakumar ${ }^{1,2}$, M. Krishna $\operatorname{Kumar}^{3}$, R. Mohan Kumar ${ }^{1}$, R. Kanagadurai ${ }^{1}$ \\ ${ }^{1}$ Department of Physics, Presidency College, Chennai-600 005, India \\ ${ }^{2}$ Department of Physics, MNM Jain Engineering College, Chennai-600 097, India \\ ${ }^{3}$ Department of Physics, Kalasalingam University, Krishnankoil-626 126, India
}

Received October 03, 2015; accepted November 24, 2015; published December 31, 2015

\begin{abstract}
Nitroanilinium 5-Sulfosalicylic acid monohydrate (4NASA) was synthesized and single crystal was grown from ethanol: water mixed solvent. The crystal system of 4NASA was determined from the single crystal X-ray diffraction study. The absorption range of 4NASA compound was analyzed from the UV-visible absorption spectral studies. The nonlinear refractive index $\left(\mathrm{n}_{2}\right)$, nonlinear absorption coefficient $(\beta)$ and third-order nonlinear optical susceptibility $\left(\chi^{3}\right)$ of 4NASA crystal were calculated by the Z-scan technique. The thermal behavior of a grown crystal was elucidated by using TG-DTA analysis.
\end{abstract}

The origin of nonlinear processes is well understood and the progress depends on the development of materials technology compatible with various device embodiments [1-2]. The ultimate goal for designing novel molecules with large third-order nonlinearities is to incorporate them into devices used in optical signal processing. The impetus for this activity has been the quest for fundamental understanding of the structure-property relationship and the strong technological interest in all optical signal processing provided by the third-order processes [3]. The 4-Nitroaniline molecule has both large hyperpolarizability $(\beta)$ and high transparency at $\lambda=532 \mathrm{~nm}$ [4]. 5-Sulfosalicylic acid has three potential coordination sites and it can give mono-, di- and tri-anionic ligand species through deprotonation. In this report, the material synthesis, crystal growth, structure, thermal, linear and third-order nonlinear optical properties of 4NASA have been presented.

The starting materials of 4-nitroaniline and 5-sulfosalicylic acid in analytical grade purity were used for the synthesis of 4NASA compound. Equimolar amounts of reagents were dissolved in ethanol and water (1:1) mixed solvent. The solution was stirred for about 6 hrs to complete the action and to get a homogeneous solution. Then, the solution was filtered to remove impurities and allow slow evaporation. The salt was collected at the bottom of a beaker and it was purified further by the recrystallization process. The solution was allowed to evaporate slowly and yielded 4NASA single crystal $\left(19 \times 9 \times 5 \mathrm{~mm}^{3}\right)$ in a period of 3-4 weeks (Fig.1).

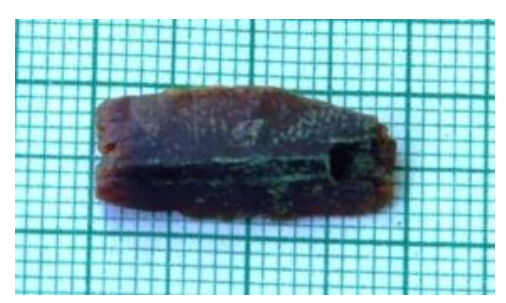

Fig.1. Photograph as grown 4NASA crystal.

Single crystal X-ray diffraction studies have been carried out for 4NASA crystal using Bruker kappa APEX II single crystal X-ray diffractometer with MoK $\alpha$ $(\lambda=0.71073 \AA)$ radiation. The calculated cell parameters are $a=13.26 \AA, b=13.55 \AA, c=17.12 \AA, \alpha=\beta=\gamma=90^{\circ}$, Volume $V=3080.23 \AA^{3}$ and $Z=8$. The grown crystal belongs to the orthorhombic crystal system with space group Pbca [5]. The unit molecule of 4NASA consists of one $\mathrm{C}_{6} \mathrm{H}_{7} \mathrm{~N}_{2} \mathrm{O}_{2}{ }^{+}$cation, one $\mathrm{C}_{7} \mathrm{H}_{5} \mathrm{O}_{6} \mathrm{~S}^{-}$anion and a water molecule in the asymmetric unit (Fig. 2). The weak intermolecular interactions through $\mathrm{N}-\mathrm{H} \cdots \mathrm{O}, \mathrm{O}-\mathrm{H} \cdots \mathrm{O}$, $\mathrm{C}-\mathrm{H} \cdots \mathrm{O}$ hydrogen bonds and $\pi-\pi$ interactions are stabilized to form a three-dimensional molecular arrangement. The dihedral angle between the two benzene rings $(\mathrm{C} 1-\mathrm{C} 6)$ and $(\mathrm{C} 8-\mathrm{C} 13)$ is found to be $8.18(7)^{\circ}$. The benzene ring $(\mathrm{C} 1-\mathrm{C} 6)$ is planar and makes a dihedral angle of $1.32(19)^{\circ}$ with an attached Nitro group. The mean plane of carboxy group is inclined at an angle of $8.76(8)^{\circ}$ with the planar benzene ring $(\mathrm{C} 8-\mathrm{C} 13)$ of an ion.

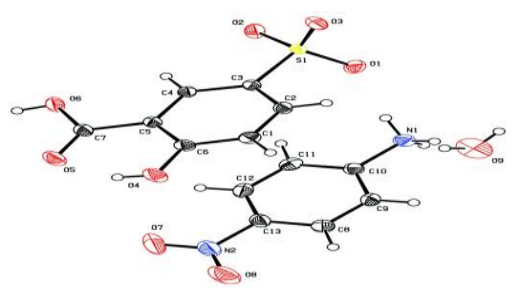

Fig. 2. Unit cell molecular structure of 4NASA. 
The linear optical absorption property was studied using a Perkin Elmer Lambda 35 UV-vis spectrophotometer. The absorption spectrum recorded for the 4NASA crystal in the region $400-700 \mathrm{~nm}$ is shown in Fig. 3. It is observed from the spectrum that the grown 4NASA single crystal has the absorption of light in the region below $437 \mathrm{~nm}$. Also, it is observed that the grown crystal has a good transparency window in the 437-700 nm range.

Its transparency lies in the violet region and the blue one, which is most interesting in optical applications.

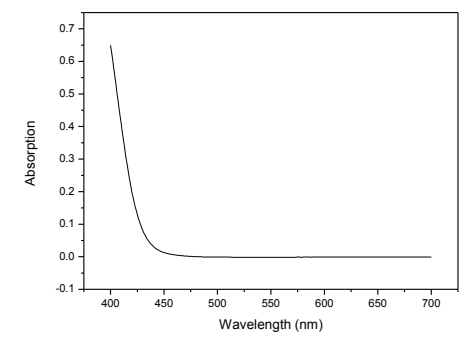

Fig. 3. UV-visible absorption spectrum of 4NASA crystal.

The Z-scan measurements are important for characterizing a third-order nonlinear optical property of materials [6]. It provides information to estimate the nonlinear refractive index $\left(\mathrm{n}_{2}\right)$, nonlinear absorption coefficient $(\beta)$ and third-order nonlinear optical susceptibility of materials [7]. For this single-beam Zscan technique, a CW laser at $632.8 \mathrm{~nm}$ (He-Ne laser) was used. The study of nonlinear refraction $\left(n_{2}\right)$ is based on the dependence on the position $z$ of the thin sample along a focused Gaussian laser beam. The power of the Gaussian beam is varied by passing it through a small aperture placed in a far field. Two different modes of interaction such as open aperture and closed aperture were studied, when a 1-mm-thick sample was moved along $z$. The "peak-valley" or "valley-peak" dependence of beam transmittance with respect to the position of sample $z$ was obtained. During this interaction, good quality laser irradiation was obtained, which indicates the optical nonlinearity of the 4NASA material. The resulting beam was studied further for identifying the nonlinearities.

Nonlinear absorption was measured in the absence of an aperture (Open-aperture Z-scan) (Fig. 4). The closed aperture Z-scan experiment was employed for 4NASA crystal (Fig. 5) by placing the aperture between the sample and power meters. The analysis of data and the determination of NLO parameters, linear absorption have been taken into account through $L_{\text {eff }}$, its contribution being successfully removed [8].

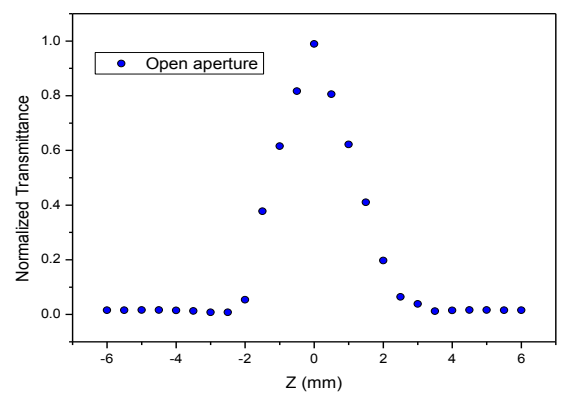

Fig. 4. Open aperture Z-scan curve of 4NASA.

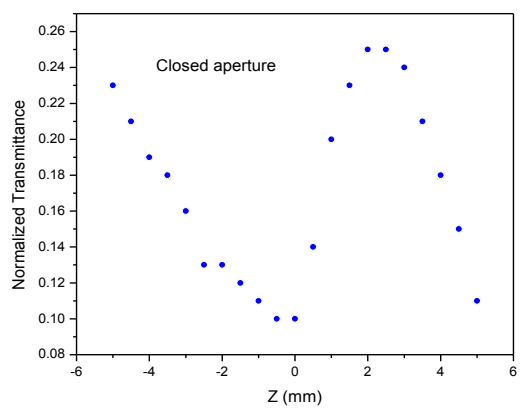

Fig. 5. Closed aperture Z-scan curve of 4NASA.

The valley followed by the peak is the signature for the nonlinear refractive index to be positive (i.e. selffocussing in nature). Further, the observed asymmetric nature of the $\mathrm{Z}$-scan measurements is due to the fact that the laser beam used in the experiment is a $\mathrm{CW}$, the valleypeak configuration suggests that the nonlinear refractive index observed is of thermal-origin. The nonlinear refractive index effect may arise from different physical mechanisms such as electronic (Kerr effect) or thermal effects (focusing and defocusing). The nonlinear refractive index for the sample in this case may be attributed to thermal nonlinearity resulting from the absorption of an incident beam by the medium, which results in the deposition of heat via non-radiative decay from excited states where a transverse temperature gradient is established due to the temperature coefficient of the refractive index $\mathrm{d} n / \mathrm{d} t$ [9].

The observed peak and valley values were used to calculate third-order nonlinear optical parameters. According to the equations described in the literature, the nonlinear optical refractive index $\left(\mathrm{n}_{2}=1.83 \cdot 10^{-11} \mathrm{~m}^{2} / \mathrm{W}\right)$ and nonlinear optical absorption coefficient $\left(\beta=1.39 \cdot 10^{-7}\right.$ $\mathrm{m} / \mathrm{W}$ ) were estimated for 4NASA [10-11]. The real and imaginary parts of the third order nonlinear optical susceptibility $\left(\chi^{3}\right)$ [12] were estimated using the following relations,

$$
\operatorname{Re} \chi^{(3)}=\frac{10^{-4} \varepsilon_{0} \mathrm{c}^{2} n_{0}^{2} n_{2}}{\pi} \text { esu }
$$




$$
\operatorname{Im} \chi^{(3)}=\frac{10^{-2} \varepsilon_{0} \mathrm{c}^{2} n_{0}^{2} \lambda \beta}{4 \pi^{2}} \quad \text { esu }
$$

where $\varepsilon_{\mathrm{o}}$ is the vacuum permittivity $\left(8.854 \cdot 10^{-12} \mathrm{~F} / \mathrm{m}\right)$, c is the velocity of light in vacuum, $n_{0}$ is the linear refractive index, $\lambda$ is the wavelength of the laser beam. The thirdorder nonlinear optical susceptibility was calculated using:

$$
\chi^{(3)}=\sqrt{\left(\operatorname{Re} \chi^{(3)}\right)^{2}+\left(\operatorname{Im} \chi^{(3)}\right)^{2}} \text { esu }
$$

The third-order nonlinear optical susceptibility is found to be $\chi^{(3)}=1.4 \cdot 10^{-7}$ esu. The third order nonlinearity data of the 4NASA crystal were compared with the reported values of some well-known NLO materials like $\mathrm{Ba}\left(\mathrm{NO}_{3}\right)_{2}$, $\mathrm{KTA}, \mathrm{MeCu}$ [13-15] and presented in Table 1. It reveals that $n_{2}$ value of 4NASA crystal is higher than the referred crystal. Due to excellent nonlinear response, 4NASA crystal can be a promising material for nonlinear optical applications.

Table 1. Comparison of Z-scan results of 4NASA crystal with some well-known NLO crystals.

\begin{tabular}{lccc}
\hline Crystal & $n_{2}\left(\mathrm{~m}^{2} / \mathrm{W}\right)$ & $\beta(\mathrm{m} / \mathrm{W})$ & References \\
\hline $\mathrm{Ba}\left(\mathrm{NO}_{3}\right)_{2}$ & $7.1 \cdot 10^{-20}$ & $21 \cdot 10^{-12}$ & {$[13]$} \\
$\mathrm{KTA}$ & $1.7 \cdot 10^{-19}$ & - & {$[14]$} \\
$\mathrm{MeCu}$ & - & $4 \cdot 10^{-13}$ & {$[15]$} \\
4NASA & $1.83 \cdot 10^{-11}$ & $1.39 \cdot 10^{-7}$ & present work \\
\hline
\end{tabular}

A simultaneous TGA/DTA measurement was carried out in the temperature range from 31 to $600^{\circ} \mathrm{C}$ in the nitrogen atmosphere at a scanning rate of $10^{\circ} \mathrm{C} / \mathrm{min}$ (Fig. 4). The TGA curve showed five distinct weight loss stages in the 4NASA compound. The first weight loss observed at $107.6^{\circ} \mathrm{C}$ is due to the loss of $\mathrm{H}_{2} \mathrm{O}$ molecules and causes about $1.24 \%$ weight loss of the compound. During the second stage up to $192.4^{\circ} \mathrm{C}$, the compound lost its weight again approximately $7.6 \%$ at $192^{\circ} \mathrm{C}$ due to further loss of $\mathrm{H}_{2} \mathrm{O}$ molecules component of 4NASA. The third decomposition stage extended up to a temperature of $343.3^{\circ} \mathrm{C}$ and the compound lost $44 \%$ of its weight at this stage. It was due to the exit of $\mathrm{NO}_{2}, \mathrm{CO}_{2}$ gases from the compound. The fourth stage of decomposition was found between $343.3-465.8^{\circ} \mathrm{C}$ and it was due to the exit of $\mathrm{SO}_{2}$. The fifth decomposition step was observed above $465.8^{\circ} \mathrm{C}$ and the benzene structure of the compound was decomposed at this stage. The residual mass of the sample was found to be $30 \%$ at the temperature of $600^{\circ} \mathrm{C}$. From the DTA curve an endothermic peak was observed at $219.4^{\circ} \mathrm{C}$ due to the melting of $4 \mathrm{NASA}$.

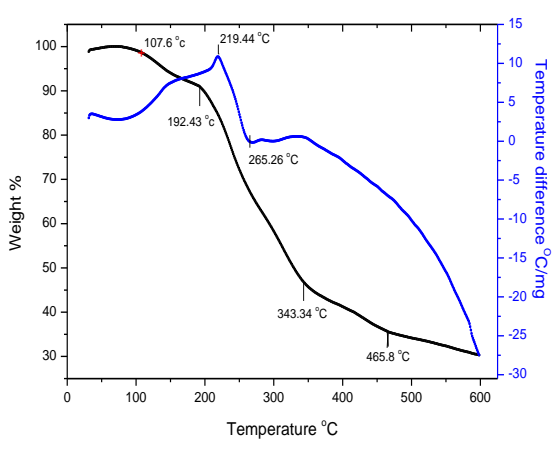

Fig. 4. TGA-DTA curve for 4NASA compound.

In conclusion, a third-order nonlinear optical single crystal 4-Nitroanilinium 5-Sulfosalicylic acid monohydrate (4NASA) was grown by the slow evaporation method.

The crystal system and cell parameters were evaluated by single crystal X-ray diffraction analysis. The optical absorption spectrum shows that 4NASA has a good optical window in the blue and visible regions.

A Z-scan technique is used to measure the nonlinear refractive index, nonlinear absorption coefficient and third-order nonlinear optical susceptibility. Thermal decomposition stages and stability of 4NASA were found.

\section{References}

[1] D.F. Eaton, Science 253, 281 (1991).

[2] R.L. Sutherland, Handbook of Nonlinear Optics (Marcel Dekker Inc, New York 2003).

[3] J.G. Breitzer, D.D. Dlott, L.K. Iwaki, S.M. Kirkpatrick, T.B. Rauchfuss, J. Phys. Chem. A 103, 6930 (1999).

[4] P.N. Prasad, D.J. Williams, Introduction to Nonlinear Optical Effects in Organic Molecules and Polymers (Wiley, New York 1991).

[5] P.K. Sivakumar, M. Krishna Kumar, G. Chakkaravarthi, R. Mohan Kumar, R. Kanagadurai, Acta Cryst. E 69, 1609 (2013).

[6] S. Anandhi, T.S. Shyju, T.P. Srinivasan, R. Gopalakrishnan, J. Cryst. Growth 335, 75 (2011).

[7] A. Chandramohan, R. Bharathikannan, M.A. Kandhaswamy, J. Chandrasekaran, R. Renganathan, V. Kandavelu, Cryst. Res. Technol. 43, 173 (2008).

[8] W. Lei, X. L.Yan, X. RuiFeng, L. XiFeng, C. Raisin, T. XuTang, Sci. China Chem. 55, 1138 (2012).

[9] F. Khammar, A.P. Kerasidou, K. Iliopoulos, P. Savel, H. Akdas- Kilig Y. Hamaizi, J.-L. Fillaut, B. Sahraoui, J. Opt. Soc. Am. B. 07, 1555 (2014).

[10] F.L.S. Cuppo, A.M.F. Neto, S.L. Gomeze, P.P. Muhoray, J. Opt. Soc. Am. B 19, 1342 (2002).

[11] M. Krishna Kumar, S. Sudhahar, A. Silambarasan, B.M. Sornamurthy, R. Mohan Kumar, Optik 125,751 (2014).

[12] M. Sheik-Bahae, A.A. Said, T.H. Wei, D.J. Hagan, E.W. Van Stryland, J. Quantum Electron. 26,760 (1990).

[13] K. Iliopoulos, I. Guezguez, A.P. Kerasidou, A-El. Ghayoury, D. Branzea, G. Nita, N. Avarvari, H. Belmabrouk, S. Couris, B. Sahraoui, Dyes and Pigments 101, 229 (2014).

[14] T.C. Sabari Girisun, S. Dhanuskodi, D. Mangalaraj, J. Phillip, Current. Appl. Phys. 11, 838 (2011).

[15] A.I. Vodchits, V.P. Kozich, V.A. Orlovich, P.A. Apanasevich, Opt. Commun. 263, 304 (2006).

[16] H.P. Li, C.H. Kam, Y.L. Lam, W. Ji, Opt. Mater. 15, 237 (2001). 